\title{
NEW HYBRID PAN-SHARPENING METHOD BASED ON TYPE-1 FUZZY-DWT STRATEGY
}

\author{
Farzaneh Dadras Javan*, Faezeh Sadat Mortazavi, Fateme Moradi, Ahmad Toosi
}

Department of Geomatics, University College of Engineering, University of Tehran, Tehran, Iran - (fdadrasjavan, faezeh.mortazavi, Fatemeh.moradii, ahmadtoosi71)@ut.ac.ir

KEY WORDS: Image Fusion, Pan-sharpening, Fuzzy, Discrete Wavelet Transform, Type-1 Fuzzy-DWT

\begin{abstract}
:
The purpose of image fusion is to combine two images from the same view in order to produce an image with more information and higher quality. In this paper, a panchromatic image with high spatial resolution and a low-resolution multi-spectral image having rich spectral information are fused together to produce a high-resolution multi-spectral image that heritage the characteristics of both initial images. For this purpose, a hybrid pan-sharpening method, called 'Improved Fuzzy-DWT' have been proposed based on the modification of the parameters existed in the latest version of Fuzzy-Wavelet algorithm, and then it was compared with some other algorithms such as PCA, Gram-Schmidt, Wavelet, and two of its hybrid derivatives called PCA-Wavelet and IHS-wavelet. The comparison was conducted using DIV, SSIM, SID, CC, Ds, and QNR spectral and spatial quality assessment metrics. The obtained results demonstrate that the proposed hybrid algorithm has relatively better performance in comparison with the other mentioned pansharpening techniques in terms of both spectral and spatial qualities, regarding it was superior in terms of SID, DIV, SSIM, Ds. From the computational cost standpoint, the proposed method has the most running time compared with the other methods.
\end{abstract}

\section{INTRODUCTION}

Nowadays, satellite imageries are applied in several fields such as agriculture, geology, urban studies, photogrammetry, remote sensing, etc (Ranchin and Wald, 2000; Reyes et al., 2004; Fonseca et al., 2011; Hasanlou and Saradjian, 2016; DadrasJavan et al., 2018). Considering the above-mentioned applications, improving the quality of satellite imageries is getting very important. Since there is no sensor to produce an image with rich spectral quality and high spatial resolution at the same time, so most of the time the initial images produced by satellites are fused together so that the output image supplies the researchers' demands (Pohl and Genderen, 1998; DadrasJavan and Samadzadegan, 2014; Jangalingam and Hegde, 2015). In photogrammetry and remote sensing application, a variety of images, such as multi-view, multi-focus, multi-sensor, multitemporal and multi-spectral images are fused with each other (Flusser et al., 2007; Yang et al., 2014; Özkan et al., 2016). According to the goal of this paper, we try to fuse the multispectral and panchromatic images to achieve a high-spatialresolution image that enjoys the high spectral information content at the same time. When multi-spectral and panchromatic images are considered as the inputs of the image fusion process, it is called 'pan-sharpening' (Vivone et al., 2015).

Panchromatic images are single-band grey level images that provide high spatial resolution and show the Earth spatial details precisely. On the other hand, multi-spectral images have low spatial resolution and high spectral quality and can show the spectral attributes of the objects (Rodriguez-Esparragon et al., 2017). As none of these two above-mentioned images can lonely provide us complete information that we need, so by fusing them, a fused image is created that heritage the spectral information from the multi-spectral image, and also its spatial details are captured from the panchromatic image, by injection the spatial characteristics from the panchromatic image to the multi-spectral data (DadrasJavan and Samadzadegan, 2014). The fusion process helps us to have better visual comprehension and better interpretation of the images by increasing the quality of the fused image in comparison with the original images (Stein, 2005).

In general, an image fusion process can be conducted at three different levels of pixels, features, and decisions (Pohl and Genderen, 1998). In pixel-level image fusion, which is the lowest level of fusion, several methods have been proposed (Pandit and Bhiwani, 2015). Discrete wavelet transform (DWT) is one of the state-of-the-art pixel-based methods which is divided under the Multi-Resolution Analysis (MRA) fusion category (Jagruti, 2014). All of the fusion methods suffer from resulting in some distortions in the generated fused images (Snehmani et al., 2017).

Some attempts have been made to solve the above-mentioned problems, to increase the efficiency of the fusion methods, and also to reduce the distortions of pan-sharpened product. Artificial intelligence-based fusion techniques, such as fuzzy logic-based algorithms, are one of those strategies which are expected to help us to improve the performance of the commonly used fusion methods. The fusion of images using fuzzy logic, due to its ability to handle uncertainties, leads to improve the quality of the outputs in comparison with the conventional fusion techniques (Singh et al., 2004; Myna and Prakash, 2018).

Up to now, a variety of related studies have been conducted in the field of pan-sharpening using the concept of fuzziness and wavelet tools (Meitzler et al., 2002; Zhu and Yang, 2008; Vivone et al., 2015; Snehmani et al., 2017; DadrasJavan et al., 2018). Swathi et al. (2013) developed a novel image fusion algorithm, which is called 'Fuzzylet', by combining the features of stationary wavelet transform (SWT) and fuzzy logic. A novel approach for performing multi-focus image fusion using fuzzy logic type-1 and wavelet transform has been proposed by Myna and Prakash (2014). Myna et al. (2015) have proposed a novel hybrid approach for fusion of CT and MRI medical images. Myna and Prakash (2018) used texture-based image fusion and combine it with interval type-2 fuzzy logic and discrete wavelet transforms. Teggihalli and Ramya (2018) presented an image fusion methodology for fusing medical images using novel discrete wavelet transform type-2 fuzzy logic system.

\footnotetext{
* Corresponding author
} 
With focusing on the Fuzzy-Wavelet image fusion strategy it can be said that generally, there are two components in this hybrid procedure namely 'wavelet transform' and 'fuzzy logic system'. As presented in Figure 1, in DWT scheme, firstly, the initial images are decomposed using a discrete wavelet transform to high and low frequencies, which represent the base general spectral and details of the images, respectively. The decomposition takes place in two levels. In fact, the images are divided into four sub-images as ' $\mathrm{LL}$ ', 'LH', 'HL', and 'HH'. The mentioned components are the image approximation coefficients. ' $L L$ ' is the coefficient that is used in the subsequent decomposition. Furthermore, 'LH', 'HL', and 'HH' represent the vertical-detail component, horizontal-detail component and the detail component along the image diameter, respectively (Myna and Prakash, 2014; Teggihalli and Ramya, 2018).

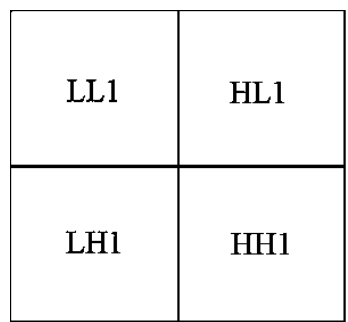

(a)

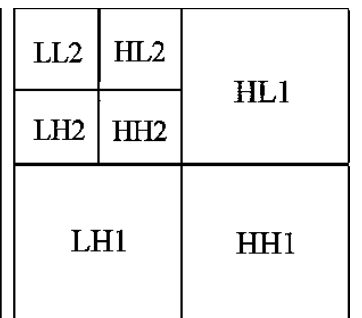

(b)
Figure 1. Image decomposition using DWT: (a) Level 1, (b) Level 2.

The fuzzy logic system is used to model uncertainties, which can not be expressed in crisp mode, with the means of expert knowledge (Myna and Prakash, 2018). Figure 2 shows the four main steps of the fuzzy inference system (FIS) which will be used in image fusion process (Guo and Wong, 2013; Kumaraswamy et al., 2016).

1. Fuzzification of input data: In this step the crisp input variables are fuzzified and a degree of membership is determined to each of them using the membership functions. In this paper, the 'Gaussian' membership function has been used.

2. Evaluation of fuzzy rules: After fuzzification of the input data and obtaining their membership values, they are applied to the antecedents of fuzzy rules. If a given fuzzy rule has multiple antecedents, the fuzzy operator (AND or OR) are used to obtain a single number that shows the result of the antecedent evaluation. This number is then applied to a consequent membership function.

3. Aggregation of outputs of fuzzy rules: In this stage, the outputs of all the fuzzy rules are unified to generate a single compound output. Aggregation takes membership functions of all rules' consequents and combines them into a single fuzzy set. In this paper, we utilized 'union' to implement aggregation.

4. Defuzzification of the output: In the last step, a crisp output is produced from the aggregated output of the fuzzy set.

The fuzzy logic technique is now being widely used for image fusion tasks (Singh et al., 2004). The fuzzy logic approaches are used where there are uncertainties and no mathematical relations

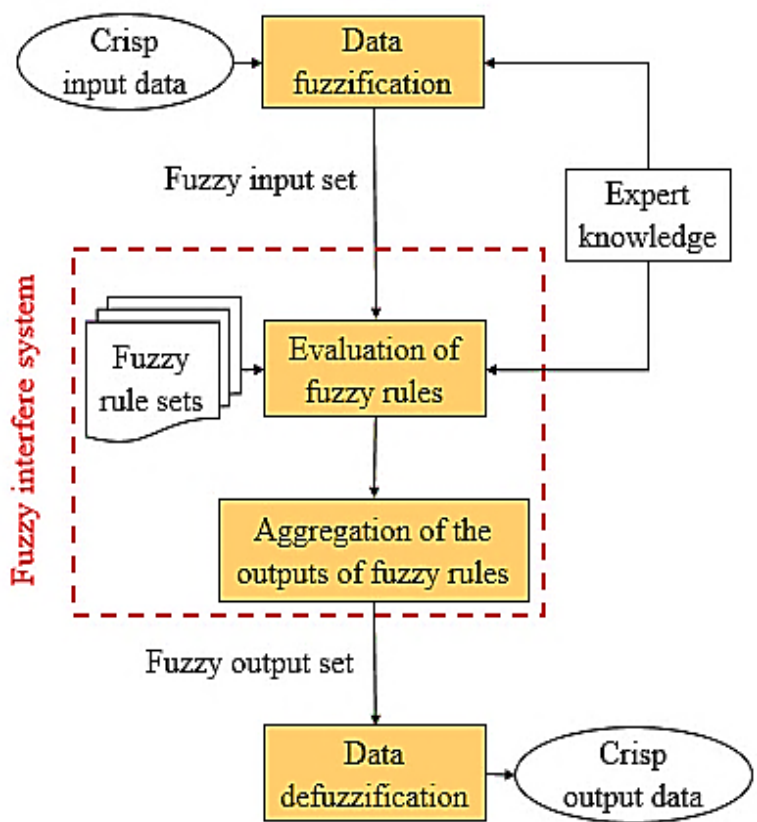

Figure 2. Flowchart of a fuzzy logic system

are easily available (Singh et al., 2004). The fuzzy logic was proposed by Zadeh (Rao et al., 2018) and is widely used in various fields. Rao et al. (2018) discovered the capabilities of image fusion using fuzzy and neuro-fuzzy methods along with eminence assessment indices. In a research that was conducted by Kumaraswamy et al. (2016), the fuzzy logic-based image fusion for satellite images obsolete conferred and the result analysis certainly proves that the proposed fuzzy logic-based fusion provides huge progress on the attainment of the process. Ramya and Sujatha (2019) presented a novel multi-modal sensor medical image fusion technique based on type-2 fuzzy logic. Seng et al. (2010) have presented a new method for pixel-wise image fusion for multi-view through-the-wall radar imaging (TWRI) that was performed based on fuzzy logic.

In this paper, a new hybrid pan-sharpening method was developed based on the combination of the two-level DWT-based image fusion and type-1 fuzzy logic-based image fusion strategy for the fusion of high-resolution Earth observation satellite imageries. The obtained results show that this modified proposed scheme improved the performance of the simple DWT method and have a relatively better spectral and spatial performance in comparison with the other studied algorithms. Improvement of the proposed method compared with the similar existed FuzzyWavelet based methods is obtained with the means of setting the best fuzzy and wavelet elements based on the similar reference papers and furthermore tuning the parameters using trial and error mechanism

\section{MATERIALS AND METHODS}

The proposed pan-sharpening method, which can be called 'New/Improved Fuzzy-DWT', uses a two-level wavelet transformation to access the details components of the initial panchromatic and multi-spectral images. As presented in Figure 3 the proposed method consists of three main stages as 'Decomposition', 'Fusion', and 'Reconstruction'. After Decomposition (which is schematically presented in Figure 1), the low-frequency general information of the panchromatic and multi-spectral images, obtained in the Decomposition stage are fused together using the averaging mechanism. We used the 
averaging method as an alternative for the maximum procedure, which was utilized by Teggihalli and Ramya (2018), as we deduced that the averaging performance is relatively better compared with the maximum method. Also, the horizontal, vertical, and diagonal detail components of two images, which was obtained using the DWT scheme, are inserted to the fuzzy process and are fused based on the pre-defined fuzzy rules and the membership functions. In this paper, the 'Mamdani' fuzzy inference system is used to fuse the details of two images. This FIS type was chosen based on the comparison which was carried out by Hamam and Georganas (2008). Based on the proposed algorithm, first, the images are decomposed using DWT, and then fused together and finally, the fused image is reconstructed with the means of inverse discrete wavelet transform (IDWT).

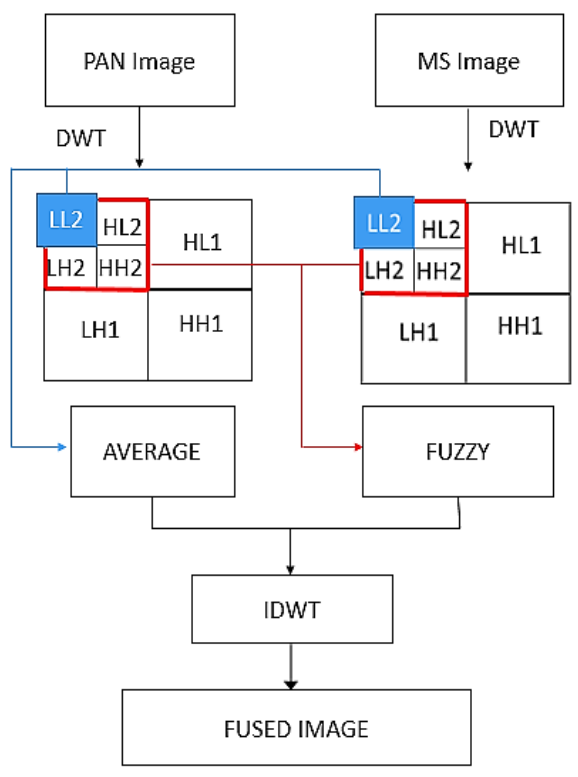

Figure 3. Diagram of proposed Fuzzy-DWT fusion algorithm

\subsection{Proposed Algorithm}

The operational procedure for implementing the proposed pansharpening method is made up of nine steps, as follow:

1. Input of initial images.

2. Resizing the multi-spectral image to the size of the panchromatic image.

3. Decomposing the input images using the two-level DWT and producing four sub-images (the images are transferred from the spatial domain to the frequency domain).

4. Fusion of the general information of images using the averaging strategy.

5. Three detail sub-images of the two images, which are in the form of matrices, are considered as the inputs of the fuzzy process.

6. Vectorization of the above-mentioned matrices.

7. Definition of the fuzzy rules and membership functions for the inputs.

8. Converting the structure of the outputs of the fuzzy process from column format to matrix format.

9. Using IDWT to transform the fused image from the frequency to the initial spatial domain.

\subsection{Fuzzy Inference System (FIS)}

In this paper, the Mamdani-type fuzzy inference system is used as it is widely accepted for capturing expert knowledge (Kaur and Kaur, 2012). This kind of FIS comprises two inputs and a single output, which the two inputs represent the pixel values of the approximates of the input multi-spectral and panchromatic images and the output represent the pixel values of the fused image. The Mamdani FIS uses 'OR' and 'AND'operators to evaluate maximum and minimum values in the rule sets, respectively. In the defuzzification stage, the output fuzzy value is defuzzified using the 'centroid' method, as it showed to be one of the most popular defuzzifiers (Naaz et al., 2011; Guo and Wong, 2013).

\subsection{Fuzzy Membership Functions}

Figure 4 presents the five Gaussian fuzzy membership functions that are used for the extraction of the output of FIS, i.e. fused image. The whole range of pixels is divided into five membership classes with linguistic labels of 'Very Low (VL)', 'Low (L)', 'Medium (M)', 'High (H)' and 'Very High $(\mathrm{VH})$ ' similar to the research that was conducted by Swathi et al. (2013).

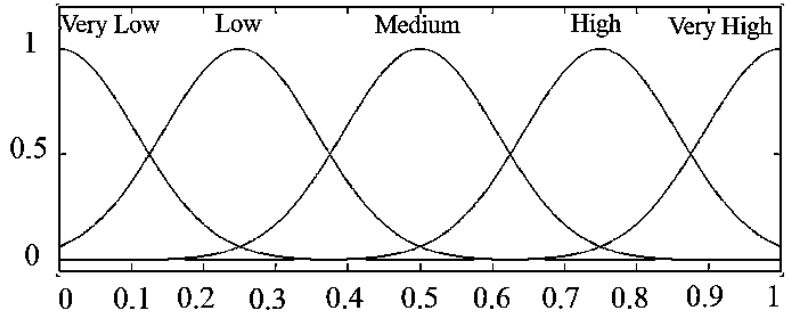

Figure 4. Using Gaussian membership functions to extract output of FIS

\subsection{Fuzzy Rules}

Fuzzy rules are used within fuzzy logic systems to infer an output based on a group of input variables. One of the forms of defining the rules is a situation in which we have a premise (for example $\mathrm{x}=\mathrm{P}$ ) and then an 'if and then' implication is defined (e.g. if $\mathrm{x}$ is $\mathrm{P}$ then $\mathrm{y}$ is $\mathrm{Q}$ ) and finally we have a consequent (like y is Q) (Enderton and Enderton, 2001). According to the Swathi et al.'s (2013) paper depending on the pixel value in both input images, the decision about the outputs is made according to Table 1 , which contains the fusion rules used in fuzzy logic for five membership functions. To clarify Table 1, suppose that a pixel in the input 1 (panchromatic image) and input 2 (multi-spectral image) have Low and High values, respectively, then the value of the corresponding pixel in the output fused image will be Medium. As we used five membership functions, then we will have $5 \times 5$, i.e. 25 , fuzzy rules in our FIS model. For example five of the fuzzy rules of the fuzzy logic systems are defined as follow:

1. If (input1 is Very Low) and (input2 is Low) then (output1 is Very Low).

2. If (input1 is Low) and (input2 is Medium) then (output1 is Low). 
3. If (input1 is Medium) and (input2 is Very High) then (output1 is High).

4. If (input1 is High) and (input2 is Very Low) then (output1 is Low).

5. If (input1 is Very High) and (input2 is Medium) then (output1 is High).

\begin{tabular}{|c|c|c|c|c|c|}
\hline \multirow{2}{*}{ Input 1 } & \multicolumn{5}{|c|}{ Input 2 } \\
\cline { 2 - 6 } & VL & L & M & H & VH \\
\hline VL & VL & VL & L & L & M \\
\hline L & VL & L & L & M & H \\
\hline M & L & L & M & H & H \\
\hline H & L & M & H & H & VH \\
\hline VH & M & H & H & VH & VH \\
\hline
\end{tabular}

Table 1: Rule table and fusion rules used in fuzzy logic for five membership Gaussian functions (Swathi et al., 2013)

\section{DATA AND RESULTS}

\subsection{Dataset}

In the implementation phase, the pan-sharpening algorithms are implemented on the WorldView-2 high-resolution satellite dataset acquired from San Fransisco. This dataset includes a panchromatic band with a spatial resolution of 0.5 meters and an eight-bands multi-spectral image (consist of coastal, blue, green, yellow, red, red edge, near-infra-red 1 and near-infra-red 2 bands) with the resolution of 2 meters. The dataset is presented in Figure 5 . The dimensions of the multi-spectral and panchromatic images are $4000 \times 4000$ and $16000 \times 16000$ pixels, respectively.

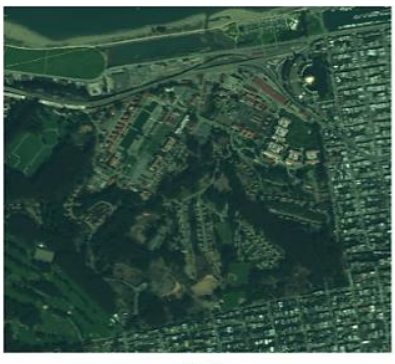

(a)

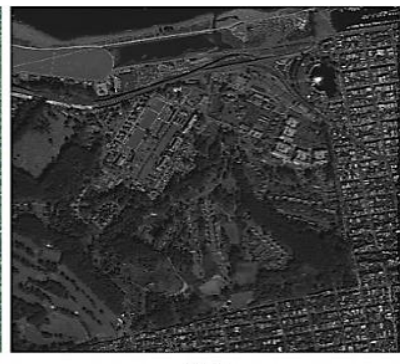

(b)
Figure 5. The San Fransisco dataset: (a) multi-spectral image, (b) panchromatic image.

\subsection{Quality Assessment Measures}

In this paper, six quality assessment metrics including four spectral metrics and two spatial metrics are applied to evaluate the spectral and spatial performance of pan-sharpening algorithms, respectively.

\subsubsection{Spectral Metrics}

Spectral Information Divergence (SID): It is used to compute the difference of the probabilistic behaviors between the normalized spectral vectors, taken from the multi-spectral and fused images ( $\mathrm{I}_{\mathrm{r}}$ and $\left.\mathrm{I}_{\mathrm{f}}\right)$ according to Equation 1. It ranges zero to 1 and the more the value is closer to 1 , more similar the images will be.

$$
\operatorname{SID}\left(I_{r}, I_{f}\right)=D\left(I_{r} \| I_{f}\right)+D\left(I_{f} \| I_{r}\right)
$$

where $D$ operator is called the relative entropy and it is defined using the spectral vectors of images (Palsson, 2013; Snehmani et al., 2017).

Correlation Coefficient (CC): This metric computes the similarity of spectral features between the multi-spectral and fused images according to Equation 2. It ranges -1 to 1 and its ideal value is 1 .

$$
C C\left(I_{r}, I_{f}\right)=\frac{\sum_{i=1}^{M} \sum_{j=1}^{N}\left[I_{r}(i, j)-\overline{I_{r}}\right]\left[I_{f}(i, j)-\overline{I_{f}}\right]}{\sum_{i=1}^{M} \sum_{j=1}^{N}\left(I_{r}(i, j)-\overline{I_{r}}\right)^{2} \sum_{i=1}^{M} \sum_{j=1}^{N}\left(I_{f}(i, j)-\overline{I_{f}}\right)^{2}}
$$

where $I_{r}(i, j)$ and $I_{f}(i, j)$ are the pixel values of the multi-spectral and fused images at position $(\mathrm{i}, \mathrm{j})$, respectively and $\overline{I_{r}}$ and $\overline{I_{f}}$ express the means of them, respectively. $M$ and $N$ are the row and column of images (Padwick et al., 2010; Yakhdani and Azizi, 2010).

Difference In Variances (DIV): It computes the quantity of information added or lost during the fusion process using Equation 3. The ideal value is zero while a positive value shows a loss of information and a negative value indicates some added information. In this paper, the positive values are considered.

$$
\operatorname{DIV}\left(I_{r}, I_{f}\right)=\frac{\sigma^{2} I_{r}-\sigma^{2} I_{f}}{\sigma^{2} I_{r}}
$$

where $\sigma_{I_{r}}^{2}$ and $\sigma^{2}{ }_{I_{f}}$ are the variances of the multi-spectral and fused images, respectively (Thomas and Wald, 2006).

Quality With No Reference (QNR): This spectral-spatial metric that is defined as Equation 4 is made up of two indices called $D_{\lambda}$ and $D_{S}$, which express the spectral and the spatial distortion of fused image, respectively. It goes zero to 1 and high value (closer to 1 ) indicates the fused image has a good quality.

$$
Q N R=\left(1-D_{\lambda}\right)^{\alpha}\left(1-D_{S}\right)^{\beta}
$$

Where $\mathrm{D}_{\lambda}$ and $\mathrm{Ds}$ are defined using Q-index; $\alpha$ and $\beta$ are constant values (Palsson, 2013; Vivone et al., 2015).

\subsubsection{Spatial Metrics}

Structural Similarity Index Metric (SSIM): This metric is used to compare the local patterns of pixel intensities between the reference and fused images according to Equation 5. Its value varies between -1 to 1 , and the more the value is closer to 1 , the more similar the reference and fused images will be.

$$
\operatorname{SSIM}\left(I_{r}, I_{f}\right)=\frac{\left(2 \mu_{I r} \mu_{I f}+C 1\right)\left(2 \sigma_{I I I f}+C 2\right)}{\left(\mu^{2} I r+\mu^{2} I f+C 1\right)\left(\sigma^{2} I r+\sigma^{2} I f+C 1\right)}
$$

where $\sigma_{I r I f}$ is covariance between the bands of reference and fused images, $\mu_{I r}\left(\mu_{I f}\right)$ and $\sigma_{I r}\left(\sigma_{I f}\right)$ are the mean and standard deviation of reference (fused) image, respectively. $C 1$ and $C 2$ are two consonant parameters (Wang et al., 2004; Kosesoy et al., 2015).

Ds: As mentioned before, the QNR metric is made up of two spectral and spatial distortion indices. The spatial distortion (Ds) index is given by Equation 6. The value of this metric varies between zero to 1 and zero is the ideal value.

$$
\mathrm{D}_{\mathrm{S}}=\frac{1}{\mathrm{n}} \sum_{\mathrm{l}=1}^{\mathrm{n}}\left|\mathrm{Q}\left(I_{f_{l}}, \mathrm{PAN}\right)-\mathrm{Q}\left(\widehat{I_{r_{l}}}-\mathrm{P} \hat{A} \mathrm{~N}\right)\right|
$$


Where $P A N$ and $I_{f_{l}}$ represent the panchromatic and fused images, respectively. Also, $\mathrm{PA} \mathrm{N}$ is the degraded panchromatic image of the same size as the multi-spectral image $\left(\widehat{I_{r_{l}}}\right)$. $Q$ refers to the Q-index, and $n$ denote the spectral bands of the image (Palsson, 2013; Vivone et al., 2015).

\subsection{Results}

For validation of the proposed pan-sharpening method, it is compared with five other methods such as Principal Component Analysis (PCA) (Fonseca et al., 2011), Gram-Schmidt (GS) (Klonus and Ehlers, 2009), Wavelet (Amolins et al., 2007), and two of the derivatives of Wavelet method, such as IHS-Wavelet (Nunez et al., 1999), and PCA-Wavelet (Gonzalez-audicana et al., 2005). The spectral quality assessment was conducted based on Wald's protocol (Ranchin and Wald, 2000); i.e. first, the input multi-spectral and panchromatic images are resampled to a lower spatial resolution and then, the resampled multi-spectral and panchromatic images are fused to generate a lower-resolution fused image and finally the fused image is compared with the original multi-spectral image (Ghassemian, 2016). In spatial case, the fused image is compared with the initial panchromatic image at its original scale. Figure 6 shows the outputs of six pansharpening methods, in addition to the initial multi-spectral and panchromatic images. In order to have a better comparison, two crops are selected from each image that are also presented. Results of the spectral and spatial quality assessment of different pan-sharpened outputs are presente in Table 2 and Table 3, respectively. The best values are bolded in order to be distinguishable.
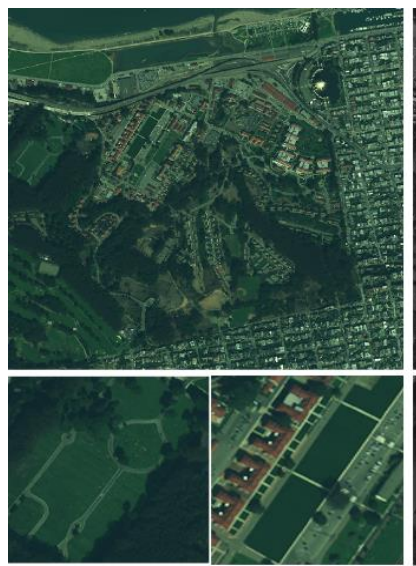

(a)

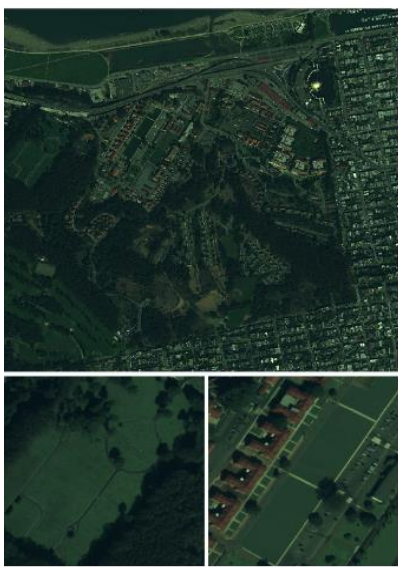

(e)
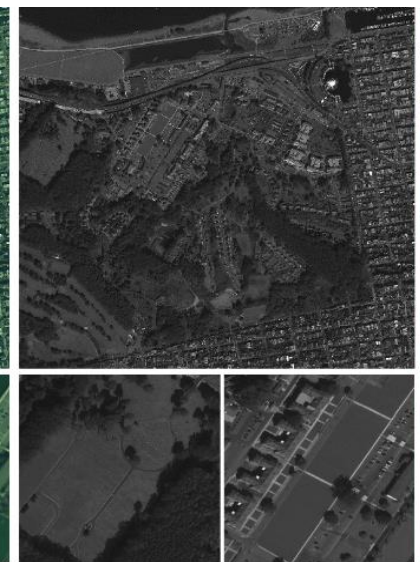

(b)

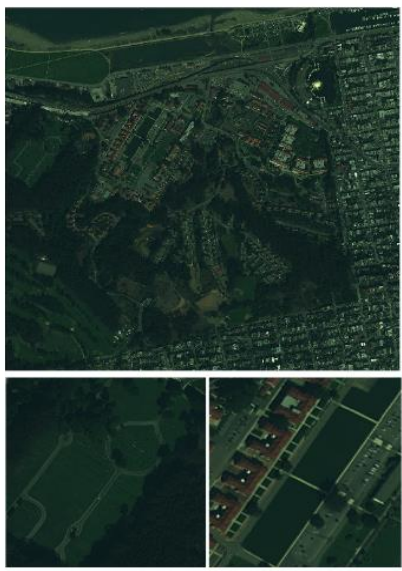

(f)

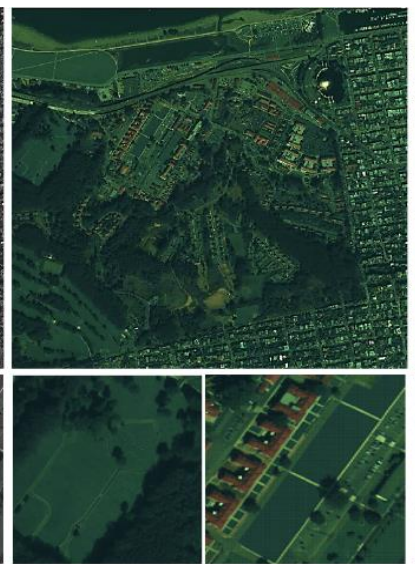

(c)

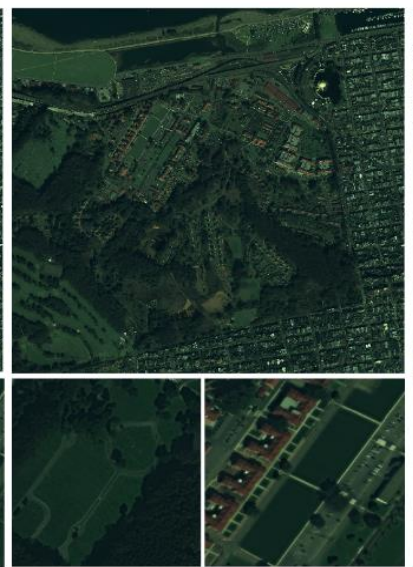

(g)

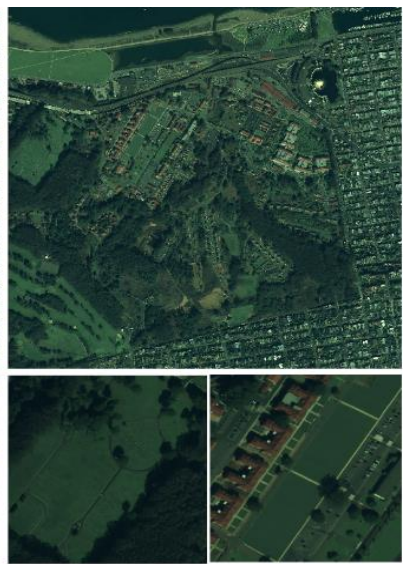

(d)

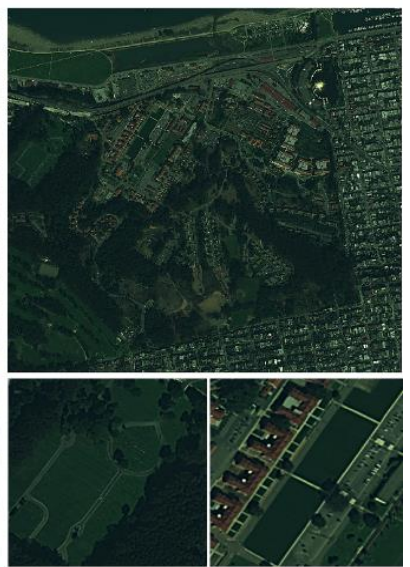

(h)

Figure 6. The inputs and outputs of pan-sharpening methods: (a) Multi-spectral, (b) Panchromatic, (c) Fuzzy-DWT, (d) PCA, (e) GS, (f) Wavelet, (g) IHS-Wavelet, (h) PCA-Wavelet.

\begin{tabular}{|c|c|c|c|c|c|c|}
\hline Metric & PCA & GS & Wavelet & IHS-Wavelet & PCA-Wavelet & Fuzzy-DWT \\
\hline SID & 0.0534 & 0.0529 & 0.0550 & 0.0498 & 0.0484 & $\mathbf{0 . 0 1 1 5}$ \\
\hline CC & 0.8705 & 0.8669 & $\mathbf{0 . 9 4 3 2}$ & 0.9385 & 0.9350 & 0.9431 \\
\hline DIV & 0.1756 & 0.1888 & 0.1823 & 0.1763 & 0.4010 & $\mathbf{0 . 1 7 2 5}$ \\
\hline QNR & 0.5069 & 0.5054 & $\mathbf{0 . 5 2 3 9}$ & 0.4151 & 0.4436 & 0.4434 \\
\hline
\end{tabular}

Table 2: Results of the spectral quality assessment of six pan-sharpening outputs 


\begin{tabular}{|c|c|c|c|c|c|c|}
\hline $\begin{array}{c}\text { Method } \\
\text { Metric }\end{array}$ & PCA & GS & Wavelet & IHS-Wavelet & PCA-Wavelet & Fuzzy-DWT \\
\hline SSIM & 0.8449 & 0.8465 & 0.8005 & 0.8255 & 0.8215 & $\mathbf{0 . 9 5 2 4}$ \\
\hline Ds & 0.5465 & 0.5484 & 0.4294 & 0.5288 & 0.5065 & $\mathbf{0 . 4 1 2 3}$ \\
\hline
\end{tabular}

Table 3: Results of the spatial quality assessment of six pan-sharpening outputs

\section{DISCUSSION AND CONCLUSION}

Image fusion (in particular, pan-sharpening) algorithms improve the quality of the initial images with the means of fusing the useful and key information of them. A variety of pan-sharpening techniques have been proposed so far. A pan-sharpening algorithm is suitable and optimum that preserve the spectral quality of the multi-spectral data well and furthermore inject the maximum possible spatial information from the panchromatic data to the fused image. This paper has focused on the development of a fuzzy-wavelet-based pan-sharpening approach. More in details in this research, a new hybrid pan-sharpening method was proposed using a type-1 fuzzy logic system and discrete wavelet transform fusion strategy to improve the performance of the former versions of Fuzzy-Wavelet-based pansharpening scheme. In this paper, first the spectral and spatial quality assessment process was conducted and then the obtained empirical result of the Fuzzy-DWT proposed pan-sharpening approach has been compared to some other state-of-the-art methods such as PCA, GS, Wavelet and the two of its derivative (hybrid) schemes called IHS-Wavelet and PCA-Wavelet. The quality assessment was conducted using four spectral quality assessment metrics such as SID, CC, DIV, QNR, and two spatial quality assessment metrics, called SID, and SSIM. The spectral evaluation was done based on the Wald's protocol and in the spatial quality assessment process, the pan-sharpened outputs were simply compared with the initial panchromatic data. All of the six pan-sharpening procedures were implemented on World view-2 high-resolution satellite imagery dataset captured from the San Fransisco state-USA.

In spectral quality point of view, regarding the outputs of spectral metrics, it is found out that the Fuzzy-DWT and after that the simple wavelet pan-sharpening algorithms had the best performance and could maintain the spectral information of the initial multi-spectral image relatively well. On the other hand, in spatial quality standpoint due to the outputs of spatial metrics, the proposed Fuzzy-DWT pan-sharpening procedure performed fairly well and could transfer the spatial details from the panchromatic image to the pan-sharpened output almost better in comparison with the other five mentioned pan-sharpening techniques.

The spectral and spatial qualities of the pan-sharpening methods are not the only factors of method selection. The computation speed and complexity of pan-sharpening algorithms are also important. Figure 7 represents the absolute time values, in minutes, spent for running each algorithm using a PC system with $2.50 \mathrm{GHz}$ Pentium Intel Core i7 Processor and 6 GB RAM. Regarding Figure 7, it is deduced that the proposed Fuzzy-DWT method has the slowest algorithm in terms of consuming time (5.133 minutes), but since this method was one of the superior methods in terms of spectral and spatial quality, it can be used in cases that the quality of the pan-sharpened image is much more important in comparison to its computational speed; i.e. this method produces a high spectral and spatial quality pansharpened image at the expense of having a relatively high

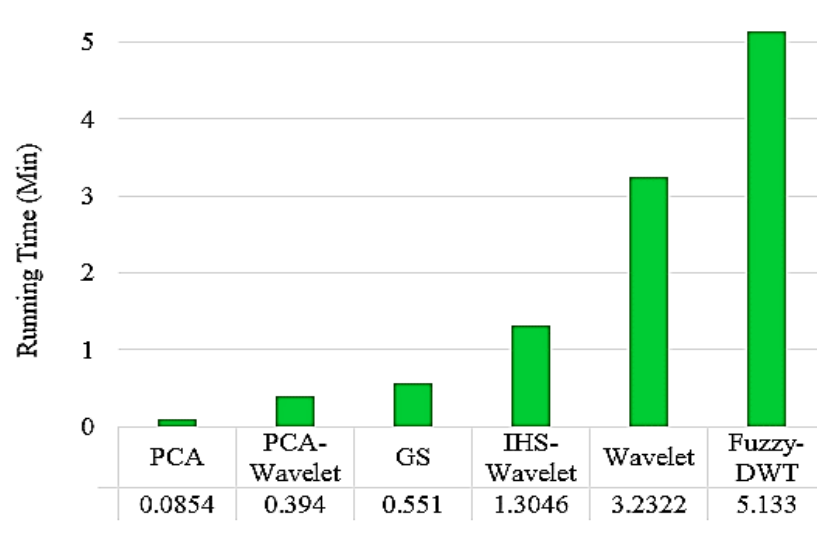

Figure 7 . The time consumed by each pan-sharpening algorithm

running time. Furthermore, the least value in terms of running time (0.0854 minutes) belongs to the PCA method.

The reason that is caused the proposed Fuzzy-Wavelet method perform remarkably better in comparison with the PCA, GS, wavelet and the two of its derivatives, was using the fuzzy inference system in the implementation of wavelet pansharpening strategy. Since the fuzzy logic system allows us to deal with issues in a non-crisp manner and have a possibility to have flexible analysis, this led to the improvement of the obtained results in both spectral and spatial quality cases. With this in mind, overall, with respect to the obtained results of this paper, it can be said that the remote-sensing and photogrammetry societies are recommended to use this proposed pan-sharpening method in their scientific programs, and even improve it if possible.

\section{REFERENCES}

Amolins, K., Zhang, Y., \& Dare, P., 2007. Wavelet based image fusion techniques-An introduction, review and comparison. ISPRS Journal of Photogrammetry and Remote Sensing, 62(4), 249-263.

DadrasJavan, F., \& Samadzadegan, F., 2014. An object-level strategy for pan-sharpening quality assessment of high-resolution satellite imagery. Advances in Space Research, 54(11), 22862295 .

DadrasJavan, F., Samadzadegan, F., \& Fathollahi, F., 2018. Spectral and Spatial Quality assessment of IHS and Wavelet Based Pan-sharpening Techniques for High Resolution Satellite Imagery. Advances in Image and Video Processing, 6(2), 01. 
Enderton, H., \& Enderton, H. B., 2001. A mathematical introduction to logic. Elsevier.

Flusser, J., Sroubek, F., \& Zitova, B., 2007. Image fusion: principles, methods, and applications. Tutorial Eusipco.

Fonseca, L., Namikawa, L., Castejon, E., Carvalho, L., Pinho, C., \& Pagamisse, A., 2011. Image fusion for remote sensing applications. In Image fusion and its applications. InTech.

Ghassemian, H., 2016. A review of remote sensing image fusion methods. Information Fusion, 32, 75-89.

González-Audícana, M., Otazu, X., Fors, O., \& Seco, A., 2005. Comparison between Mallat's and the 'à trous' discrete wavelet transform based algorithms for the fusion of multispectral and panchromatic images. International Journal of Remote Sensing, 26(3), 595-614.

Guo, Z., \& Wong, W., 2013. Fundamentals of artificial intelligence techniques for apparel management applications. Optimizing Decision Making in the Apparel Supply Chain Using Artificial Intelligence (AI): from Production to Retail, 13-40.

Hamam, A., \& Georganas, N. D., 2008, October. A comparison of Mamdani and Sugeno fuzzy inference systems for evaluating the quality of experience of Hapto-Audio-Visual applications. In 2008 IEEE International Workshop on Haptic Audio visual Environments and Games (pp. 87-92). IEEE.

Hasanlou, M., \& Saradjian, M. R., 2016. Quality assessment of pan-sharpening methods in high-resolution satellite images using radiometric and geometric index. Arabian Journal of Geosciences, 9(1), 45.

Jagalingam, P., \& Hegde, A. V., 2015. A review of quality metrics for fused image. Aquatic Procedia, 4, 133-142.

Kaur, A., \& Kaur, A., 2012. Comparison of mamdani-type and sugeno-type fuzzy inference systems for air conditioning system. International journal of soft computing and engineering, 2(2), 323-325.

Klonus, S., \& Ehlers, M., 2009, July. Performance of evaluation methods in image fusion. In Information Fusion, 2009. FUSION'09. 12th International Conference on (pp. 1409-1416). IEEE.

Kosesoy, I., Cetin, M., \& Tepecik, A., 2015. A toolbox for teaching image fusion in matlab. Procedia-Social and Behavioral Sciences, 197, 525-530.
Kumaraswamy, S., Srinivasa Rao, D., \& Naveen Kumar, N., 2016. Satellite image fusion using fuzzy logic. Informatica $8(2)$, 241-253.

Meitzler, T. J., Bednarz, D., Sohn, E. J., Lane, K., \& Bryk, D., 2002. Fuzzy logic based image fusion (No. TACOM-TP-13818). ARMY TANK-AUTOMOTIVE COMMAND WARREN MI.

Myna, A. N., \& Prakash, J., 2014. A novel hybrid approach for multi-focus image fusion using fuzzy logic and wavelets. International Journal of Emerging Trends and Technology in Computer Science,(IJETTCS), 3, 131-138.

Myna, A. N., \& Prakash, J., 2015. Fusion of CT and MRI Images Based on Fuzzy Logic and Discrete Wavelet Transform. International Journal of Computer Science and Information Technologies (IJCSIT). 6 (5), 4512-4519.

Myna, A. N., \& Prakash, J., 2018. Texture Based Multifocus Image Fusion Using Interval Type 2 Fuzzy Logic. Computer Engineering and Intelligent Systems. 9(2). ISSN (Paper)22221727 ISSN (Online)2222-2863

Naaz, S., Alam, A., \& Biswas, R., 2011. Effect of different defuzzification methods in a fuzzy based load balancing application. International Journal of Computer Science Issues (IJCSI), 8(5), 261.

Nunez, J., Otazu, X., Fors, O., Prades, A., Pala, V., \& Arbiol, R., 1999. Multiresolution-based image fusion with additive wavelet decomposition. IEEE Transactions on Geoscience and Remote sensing, 37(3), 1204-1211.

Özkan, K., Işık, Ş., \& Dordinejad, G. G., 2016, August. Multispectral image fusion based on the common vector approach. In 2016 International Symposium on INnovations in Intelligent SysTems and Applications (INISTA) (pp. 1-6). IEEE.

Pálsson, F., 2013. Pansharpening and Classification of Pansharpened Images (Doctoral dissertation).

Padwick, C., Deskevich, M., Pacifici, F., \& Smallwood, S. (2010, April). WorldView-2 pan-sharpening. In Proceedings of the ASPRS 2010 Annual Conference, San Diego, CA, USA(Vol. 2630).

Pandit, V. R., \& Bhiwani, R. J., 2015. Image fusion in remote sensing applications: A review. International journal of computer applications, 120(10).

Pohl, C., \& Van Genderen, J. L., 1998. Review article multisensor image fusion in remote sensing: concepts, methods and applications. International journal of remote sensing, 19(5), 823-854.

Ramya, H. R., Sujatha, B. K, 2019, May. Fine grained medical image fusion using type-2 fuzzy logic. Indonesian Journal of Electrical Engineering and Computer Science. 14(2). 999-1011.

Ranchin, T., \& Wald, L., 2000. Fusion of high spatial and spectral resolution images: the ARSIS concept and its implementation. Photogrammetric Engineering and Remote Sensing, 66(1), 49-61.

Rao, D. S., Rao, P. C. Y., \& Minni, S. Y., 2018. Performance Evaluation of Fuzzy and Neuro Fuzzy Based Image Fusion. 
Reyes, R. A., Gutierrez, M. J., Fernandez, S., Thomas, C., Ranchin, T., \& Wald, L., 2004, May. Evaluation of the quality of Quickbird fused products.

Rodríguez-Esparragón, D., Marcello, J., Eugenio, F., GarcíaPedrero, A., \& Gonzalo-Martín, C. , 2017. Object-based quality evaluation procedure for fused remote sensing imagery. Neurocomputing, 255, 40-51.

Seng, C. H., Bouzerdoum, A., Tivive, F. H. C., \& Amin, M. G., 2010, December. Fuzzy logic-based image fusion for multi-view through-the-wall radar. In 2010 International Conference on Digital Image Computing: Techniques and Applications (pp. 423-428). IEEE.

Singh, H., Raj, J., Kaur, G., \& Meitzler, T., 2004, July. Image fusion using fuzzy logic and applications. In 2004 IEEE International Conference on Fuzzy Systems (IEEE Cat. No. 04CH37542) (Vol. 1, pp. 337-340). IEEE.

Snehmani, Gore, A., Ganju, A., Kumar, S., \& Srivastava, P. K., 2017. A comparative analysis of pansharpening techniques on QuickBird and WorldView-3 images. Geocarto International, 32(11), 1268-1284.

Stein, A., 2005. Use of single-and multi-source image fusion for statistical decision-making. International journal of applied earth observation and geoinformation, 6(3-4), 229-239.

Swathi, N., Bindu, E., \& Naidu, V. P. S., 2013. Pixel level image fusion using fuzzylet fusion algorithm. International Journal of Advanced Research in Electrical, Electronics and Instrumentation Engineering, 261-269.

Teggihalli, M., Ramya, R., 2018, May. Image Fusion Based on DWT Type-2 Fuzzy Logic System. International Journal of Scientific \& Engineering Research. 9(5).

Thomas, C., \& Wald, L., 2006, July. Analysis of changes in quality assessment with scale. In 2006 9th International Conference on Information Fusion (pp. 1-5). IEEE.

Vivone, G., Alparone, L., Chanussot, J., Dalla Mura, M., Garzelli, A., Licciardi, G. A., ... \& Wald, L., 2015. A critical comparison among pansharpening algorithms. IEEE Transactions on Geoscience and Remote Sensing, 53(5), 25652586.

Wang, Z., Bovik, A. C., Sheikh, H. R., \& Simoncelli, E. P., 2004. Image quality assessment: from error visibility to structural similarity. IEEE transactions on image processing, 13(4), 600612 .

Yakhdani, M. F., \& Azizi, A., 2010. Quality assessment of image fusion techniques for multisensor high resolution satellite images (case study: IRS-P5 and IRS-P6 satellite images) (pp. 205-209). na.

Yang, Y., Huang, S., Gao, J., \& Qian, Z., 2014. Multi-focus image fusion using an effective discrete wavelet transform based algorithm. Measurement science review, 14(2), 102-108.
Zhu, M., \& Yang, Y., 2008, October. A new image fusion algorithm based on fuzzy logic. In 2008 International Conference on Intelligent Computation Technology and Automation (ICICTA) (Vol. 2, pp. 83-86). IEEE. 